\title{
GRAN MINERÍA AURÍFERA Y SUS REPERCUSIONES EN EL DESARROLLO ECONÓMICO LOCAL*
}

\author{
Julio López Mas**** \\ E-mail: johans_jlopez@yahoo.com \\ Pablo Condori Luna**** \\ E-mail: pcondoril@gmail.com
}

\begin{abstract}
RESUMEN
Nuevas prácticas empresariales están siendo utilizadas por las empresas mineras en el Perú. La actividad minera tiene una serie de impactos microeconómicos (mejora de los ingresos, cambios en las condiciones de vida), macroeconómicos (ingreso de divisas, empleo, etc.), sociales y ambientales, los cuales pueden constituir fuente de conflicto si es que no se armoniza a la empresa y sus procesos productivos con las comunidades poblacionales localizadas en el entorno inmediato. De la misma forma, es necesario comprender que la empresa es uno de los múltiples actores en el territorio, actores que poseen diferentes intereses y capacidad de negociación; por ello se debe entender que la articulación de la empresa en el desarrollo económico local supone la adopción de políticas para mitigar los posibles impactos negativos. La experiencia de la empresa minera Barrick en su relación con la comunidad de Jangas en Ancash, es un caso característico de este fenómeno.
\end{abstract}

Palabras Clave: Responsabilidad Social Empresarial, Desarrollo Económico Local, gran minería.

\begin{abstract}
New corporate practices are being used by mining companies in Perú. Mining activity has related microeconomic impacts (income improvements, life condition changes), macroeconomic (foreing currency income, employment, etc.) social and environmental, which can constitute a conflict source if do not harmonize the corporation and its productive processes with communities settled in the inmediate environment. In the same way, it is necessary to understand that corporates are one of several performers in the territory, actors that possesses different interests and negotiation skills, for that reason is necessary be understanded that articulation of corporates in the local economic development supposes the adoption of policies to mitigate the possible negative impacts. The experience of Barrick Mining Company and its relationship with the Jangas community in Ancash is a characteristic example of this phenomenon.
\end{abstract}

Key words: Corporate social responsibility, local economic development, great miner.

\footnotetext{
* Artículo elaborado en base a la Disertación presentada en el V Encuentro Científico Internacional de Invierno. Lima, agosto del 2006.

*** Doctor en Economía e Historia Latinoamericana. Profesor Principal, Docente Investigador de la Unidad de Investigaciones y Jefe de la Oficina de Calidad y Acreditación de la Facultad de Ciencias Administrativas (UNMSM).

䊎 Licenciado en Administración de la Facultad de Ciencias Administrativas (UNMSM).
} 


\section{INTRODUCCIÓN}

Los resultados de la minería en el último quinquenio han superado las expectativas más optimistas de los inversores, funcionarios gubernamentales y sociedad en general; en ese sentido, la actividad minera metalúrgica es cada vez más relevante en su contribución al desarrollo económico del Perú. El Estado, como gestor de la política económica nacional, ha promovido sucesivamente el escenario para la incorporación de nuevas inversiones mediante mecanismos diversos, tales como contratos de estabilidad tributaria; exoneración del Impuesto a la Renta para la reinversión de utilidades, el otorgamiento de concesiones mineras, etc.; elementos que han permitido la aparición del denominado boom minero. El interés del Estado en promover la actividad minera se observó en la privatización de empresas estatales (Centromín, HierroPerú, Minero Perú, etc.); y en la exploración y explotación de nuevos yacimientos por parte de empresas particulares. Entre estos últimos encontramos a Yanacocha (1992) y Pierina (1998), ambos dedicados a la extracción de oro, y Antamina (1998), para el caso de la extracción cuprífera.

Los impactos que involucra su campo de acción son positivos en las proyecciones de largo y mediano plazo: en el nivel macro como foco de atracción de la inversión extranjera directa en el país y generador de divisas para la economía; en el nivel meso como formación de un polo económico dinámico en las zonas de influencia de los yacimientos, sean de La Libertad, Ancash, Cajamarca como casos representativos; y en el nivel micro como generador de empleo directo e indirecto, así como la mejora del nivel de ingreso y del nivel de vida de la población involucrada. Sin embargo, la experiencia histórica de la minería nacional indica que dicha explotación de minerales a la vez que impactos positivos como los mencionados, también genera impactos negativos en lo económico (fluctuaciones del tipo de cambio, exceso de divisas, desplazamiento de la mano de obra agrícola hacia la actividad minera, creación de enclaves de la actividad económica alrededor de la minería, etc.) como en lo ecológico (conflictos ambientales, uso indiscriminado de fuentes de agua, deterioro de los hábitat y de la calidad de vida de la población).

En una contribución anterior en la revista Gestión en el Tercer Milenio, hablábamos sobre la relación existente entre el desarrollo sustentable y la responsabilidad social de las empresas mineras, vinculando la investigación del caso de la minera Barrick Misquichilca S.A., la cual es una sucursal de la empresa canadiense Barrick Gold, la tercera empresa mundial de oro y que explota yacimientos a nivel internacional, con minas en operación y proyectos en desarrollo en los Estados Unidos, Canadá, Australia, Perú, Chile, Argentina y Tanzania.

Pierina, la mina donde se desarrollan las operaciones de Barrick Misquichilca S.A. (en adelante Barrick), es una mina a tajo abierto que se ubica en la cordillera de Los Andes, del Departamento de Ancash, a $10 \mathrm{~km}$ al NE de Huaraz. Está a una altitud cercana a los $4100 \mathrm{msnm}$. Inició su producción en 1998 con una inversión de US\$ 275 millones, actualmente emplea a 430 trabajadores, de los cuales más del $98 \%$ es peruano. Además hay un grupo de 450 campesinos provenientes de zonas aledañas que se desempeñan de manera rotativa en actividades complementarias, tales como reforestación, control de erosión y obras de desarrollo para sus propias comunidades. Es la segunda mina de oro más grande del país. Su producción sumada a la de Yanacocha (Cajamarca), ha hecho de Perú el primer productor de oro de Sudamérica y el octavo a nivel mundial.

La empresa Barrick al igual que Yanacocha (que como mencionamos anteriormente es la primera empresa a nivel nacional), pertenece a esta nueva generación de inversiones internacionales que poseen una visión que compromete su participación con la armonización del medio ambiente, así como con las sociedades cercanas al yacimiento, de manera que realiza su actividad económica en armonía al entorno.

Esta visión que involucra el uso racional de los recursos naturales (la mitigación de las externalidades y la armonía con las sociedades) se define como Responsabilidad Social Empresarial, la cual se entiende como una estrategia corporativa, que engloba al trinomio de la responsabilidad social (interés económico-ambiental-social) y del desarrollo sostenible (empresaambiente-sociedad), sin dejar de cumplir con sus accionistas ni con sus clientes. (Ver Gráfico 1).

Empresas como Barrick se ven obligadas -por razones de mercado y competitividad o por la existencia de normas legales- a la ejecución 


\section{Gráfico 1}

Responsabilidad social empresarial.

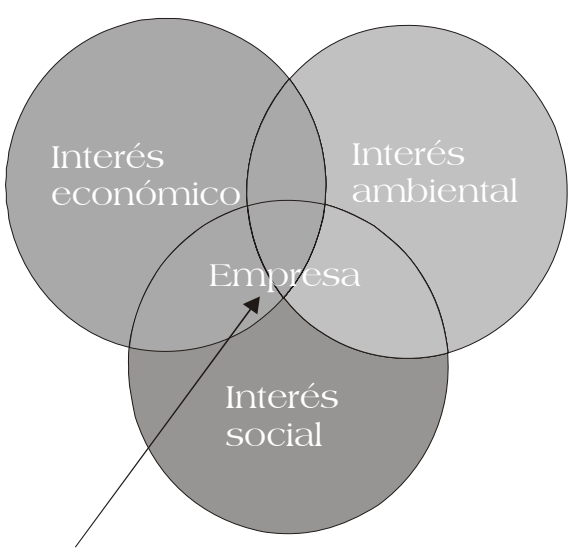

Ámbito de la

responsabilidad social

de medidas de responsabilidad social; en particular, el caso de Barrick en su relación con la comunidad de Jangas donde se encuentra ubicado el yacimiento minero.

A continuación analizaremos algunos puntos importantes que relacionan la relevancia de este concepto aunado al desarrollo local, para la comprensión integral de la relación empresaambiente-sociedad que involucra mucho más que el beneficio económico de la empresa, como la percepción que tienen las comunidades sobre el impacto de las decisiones de la empresa sobre su comunidad.

\section{DEFINICIONES PREVIAS}

\section{Responsabilidad Social}

El papel de la empresa dentro de la sociedad responde, en primer lugar, a su razón de ser: la maximización del beneficio para los propietarios (según los economistas neoclásicos, la principal responsabilidad consiste en ganar dinero para los accionistas). La empresa como tal es una construcción de nuestra sociedad moderna, y en el Perú la empresa privada se desarrolla fundamentalmente en el siglo XX; sin embargo, la óptica costobeneficio del empresariado en general se contrapone, en muchos casos, con el bienestar de las comunidades donde se realizan sus operaciones e impacta positiva o negativamente en ellas.

La Responsabilidad Social, como concepto, se puede entender como una respuesta y una necesidad de mantener el bienestar colectivo de la sociedad más allá de la simple obtención de uti- lidades; involucra como tal no solo el comportamiento de las empresas (que son, sin embargo, las principales involucradas) sino también de la comunidad y el Estado. La responsabilidad social busca además una relación estable entre los entornos, interno (trabajadores, propietarios, etc.) y externo (comunidad, clientes, proveedores y sociedad en general).

\section{Desarrollo Local y Derechos de Propiedad}

Sobre el Desarrollo Local nos ceñimos a las modernas definiciones elaboradas acerca del desarrollo económico endógeno. En una visión tradicional, se trata de impulsar el desarrollo de una comunidad en base a acciones externas (sean inversión privada o decisiones de presupuesto y normatividad públicas). En la actualidad se pone énfasis en el aprovechamiento de las capacidades, recursos y potencialidades endógenas, como recursos de la localidad o del territorio, entendidos como un nuevo punto de partida para el desarrollo desde la perspectiva local.

El tema del desarrollo local tiene una serie de matices a considerar, como lo hace Albuquerque (2003), al referir lo siguiente:

- Desarrollo local no es únicamente desarrollo municipal. El sistema productivo local, que incluye entre otros elementos el conjunto de relaciones y eslabonamientos productivos y comerciales relevantes para explicar la eficiencia productiva y competitividad de la base económica de un determinado territorio, no tiene porqué coincidir con las fronteras o delimitaciones administrativas de un municipio o provincia.

Desarrollo local no es sólo desarrollo endógeno. Muchas iniciativas de desarrollo local se basan también en el aprovechamiento de oportunidades de dinamismo exógeno. Lo importante es saber "endogeneizar» dichas oportunidades externas dentro de una estrategia de desarrollo decidida localmente.

- El desarrollo local es un enfoque territorial y de "abajo-arriba", pero debe buscar también intervenciones de los restantes niveles decisionales del Estado (provincia, región y nivel central), que faciliten el logro de los objetivos de la estrategia de desarrollo local. Se precisa, pues, de una eficiente coordinación de los diferentes niveles territoriales de las administraciones públicas y de un con- 
texto integrado coherente de las diferentes políticas de desarrollo entre esos niveles. Las decisiones de «arriba-abajo» son también importantes para el enfoque del desarrollo local.

Asimismo, hay que añadir que el desarrollo local no se limita exclusivamente al desarrollo económico local. Se trata de un enfoque integrado en el cual deben considerarse igualmente los aspectos ambientales, culturales, sociales, institucionales y de desarrollo humano del ámbito territorial respectivo ${ }^{1}$.

Aunque también Albuquerque (2004) señala que el desarrollo económico local:

«depende esencialmente de la capacidad para introducir innovaciones al interior de la base productiva y tejido empresarial de un territorio, [y que a veces], se tiene una visión muy lineal del desarrollo como si dependiera del dinamismo de los grupos empresariales [y que al tratar el tema regional] se interesa más por la posible convergencia o divergencia de las situaciones /como los indicadores entre una región $u$ otral que por entender las potencialidades de cada territorio» ${ }^{2}$.

Tanto la perspectiva de la responsabilidad social - empresarial como el desarrollo local no buscan que sea la empresa la que solucione los problemas nacionales, ni que sustituya al Estado en sus deberes, como señala la Dra. María Elena Guerra Cerrón (2001), sino que:

"...asuman un compromiso, y utilizando las herramientas de gestión empresarial, contribuyan en la búsqueda de soluciones para evitar una escalada de conflictos....”.

La existencia de derechos de propiedad en la mediana y gran minería está implícita en la dimensión microeconómica del problema, tanto en la manifestación de los conflictos como en la problemática de la posible agudización de la pobreza a causa de la sobreexplotación minera. Algunos efectos observables en este sentido son los impactos negativos mencionados por Echave y Torres (2000), tales como daños ambientales (contaminación, ruido, movimientos de tierra, etc.), salud y desarrollo humano (hacinamiento, falta de educación e información sobre enfermedades contagiosas, emigración), sobreexplotación de los recursos (muy en particular del agua) y el cierre de las minas (que no significa necesariamente la finalización de la fuente de contaminación) ${ }^{4}$.
¿Por qué incidir además en la definición apropiada de los derechos de propiedad en la inversión minera al hablar de la responsabilidad social de las empresas? Porque su no establecimiento constituye en esencia la fuente de conflicto entre las poblaciones aledañas a las zonas de extracción y las empresas, y es así que se generan exigencias ante la imposición de la inversión minera como una forma de compensar, aunque no explícitamente, la pérdida económica que constituye un desentendimiento de las empresas mineras de la comunidad.

Otro tema importante es el establecimiento de obligaciones para las empresas mineras como es el caso de las regalías, que en opinión de economistas como Iguiñiz (2003), representan una compensación frente al desgaste y al agotamiento de los recursos naturales, y que no responde necesariamente a una obligación de carácter tributario, aunque su cobro pueda ser administrado por SUNAT. Esto se fundamenta en la circunstancia de que al agotarse el mineral, éste se debe descontar como la pérdida de un activo $^{5}$. Muchos economistas no comparten esta visión y fundamentan su opinión en que las regalías mineras así como las sobreganancias representan una modificación de las denominadas reglas de juego estables que, como mencionamos al iniciar esta discusión, fueron expresadas con el ánimo de favorecer y fomentar el ingreso de la inversión privada y no necesariamente ante la previsión de un agotamiento o sobreexplotación de los recursos de las comunidades involucradas.

\section{Ley General de Minería, tributación y Canon Minero}

La Ley actualmente vigente, Ley General de Minería, fue promulgada en 1992 y posteriormente, mediante el D.S. 014-92-EM se aprobó el Texto Único Ordenado (TUO). Su Reglamento se promulgó en 1994 mediante el D.S. 03-94EM. En estos dispositivos se contemplan una serie de beneficios tributarios con el objeto de promover la inversión privada: en el Artículo 72으 del TUO se dispone que estabilidad tributaria, cambiaria y administrativa, deducción de tributos internos cuando se exporte o se venda internamente a precios internacionales, y las inversiones en infraestructura de servicios públicos, serán deducibles de la renta imponible, además de tener libertad de remisión de utilidades, di- 
videndos, recursos financieros, simplificación administrativa y silencio administrativo positivo. El valor de adquisición de las concesiones se amortizará cuando esté obligado a una producción mínima, en un plazo calculado en base a la vida probable del yacimiento. También se incluye en este beneficio lo invertido en prospección y exploración hasta cuando tenga que cumplir con una producción mínima y podrá deducirse en el ejercicio de los gastos.

La tributación minera incluye los siguientes elementos: Impuesto General a las Ventas (IGV), impuesto del $19 \%$ por la compra de bienes y servicios; Impuesto a la Renta que se determina al finalizar el año, aplicando a la utilidad una tasa de $30 \%$. En cuanto a la depreciación se aplican los siguientes supuestos: método de línea recta (3\% anual) a edificios y construcciones.

Los contratos de estabilidad tributaria por quince años implican, por su parte, cobertura, contabilidad en moneda extranjera, estabilidad tributaria, depreciación tasa global de $20 \%$, igualdad cambiaria, libre comercialización de los productos mineros y estabilidad de los regímenes especiales.

La empresa Barrick, bajo el amparo de su contrato de estabilidad tributaria, no paga regalías a pesar de existir un fallo judicial que señala que éstas no son consideradas como impuestos; en efecto, al igual que otras empresas de la gran minería que, si bien es cierto pagan fuertes cantidades en soles, éstas no son proporcionales al inesperado aumento en los precios de los minerales. Como vemos en el Gráfico 2, el precio del oro durante el periodo 2006 ha alcanzado picos que han sorprendido a propios y extraños. Durante el año 2005 y a inicios del 2006, se discutía el tema de los impuestos a las sobreganancias, y que motivaron un arduo debate acerca de la importancia del aporte de las empresas mineras al desarrollo local; todo ello concluyó al finalizar la campaña electoral y con la posterior asunción del presidente Alan García al poder en Julio del 2006, quien propuso como medida alternativa la conformación de un fondo con el aporte anual de US\$ 500 millones de dólares de las mineras para un período de cinco años, cuya administración está siendo discutida entre los aportantes, el Estado y los beneficiarios (estos últimos representados por los gobiernos locales).
Gráfico 2

Cotización del oro Ene. 2004- Dic. 2006

(US\$ por Oz Ty).

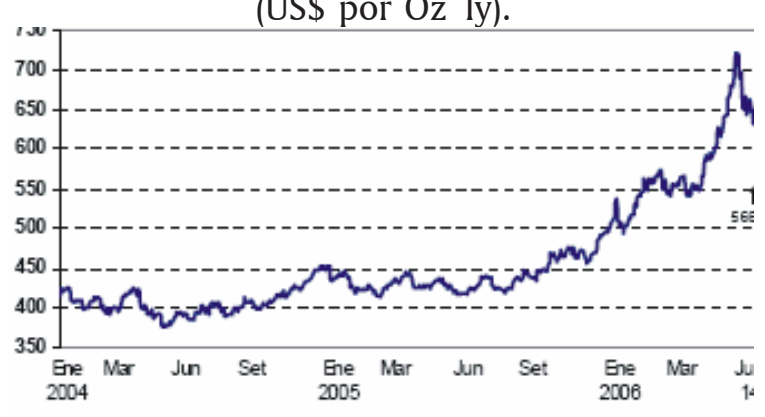

\section{OBJETIVOS}

Es interés de la investigación establecer aquellas relaciones reales entre las empresas mineras (en el ejemplo de la experiencia de Barrick, que explota el yacimiento de Pierina) y las poblaciones circundantes a las operaciones de Pierina en Huaraz (en el caso, representado por la comunidad de Jangas y alrededores en el departamento de Ancash), que son comunidades rurales con economías de supervivencia caracterizadas, al igual que otras zonas del Perú rural, por encontrarse en plena crisis de la agricultura alto andina, con altos índices de mortalidad y morbilidad, bajos niveles de alfabetismo, etc. Es importante conocer además, cuáles son las relaciones de poder y cooperación que se pueden dar entre estos dos actores y cuáles son las relaciones que efectivamente se dan.

La interrelación rápida entre la capacidad de negociación, el acceso a la información, la disponibilidad de recursos y la agilidad de la toma de decisiones, está a favor de la actividad minera, más aún cuando el Estado no está presente o lo está en forma muy débil, segmentada y parcializada, por lo que la asimetría es evidente. Cabe reconocer que en algunas provincias del Perú, la autoridad de los gobiernos locales no coincide con la autoridad del gobierno central, lo que también hace que persista el conflicto dentro del ámbito de la administración del Estado. Estas diferencias entre empresa - comunidad y gobierno local - gobierno central, impiden pensar en relaciones equitativas con tan grandes diferencias, de ahí que no basta la fría y necesariamente objetiva mirada del científico social, sino que además es necesaria -para comprender mejor la dialéctica de las relaciones entre estos actores- una mirada viva y comprometida con el proceso ineludible de empoderamiento del actor con menos capacidades de- 
sarrolladas, para que en realidad el recurso natural -el mineral aurífero- que por definición es no renovable, sea una oportunidad para el desarrollo de los pueblos y no sólo redunde en beneficio de la empresa.

Como un primer objetivo de investigación propondremos la identificación de las políticas de preservación del medio ambiente que pone en práctica la empresa Barrick dentro de su zona de influencia. Entiéndase que este conjunto de políticas debe estar enmarcado dentro de la legislación nacional, a través del cumplimiento de sus Programas de Adecuación Medioambiental (PAMA) que la empresa debe presentar al Ministerio de Energía y Minas. También es necesario identificar, dentro de las políticas de Responsabilidad Social Empresarial, el conjunto de programas sociales que la empresa pone en marcha o financia en conjunto con las autoridades locales, dentro de las zonas de influencia del yacimiento.

Como segundo objetivo es también necesario el análisis de las relaciones y la participación de la empresa Barrick con los actores locales de la sociedad. Entiéndase que existen diferentes niveles de gobierno (centro poblado, distrital, provincial, regional y nacional), cada uno con sus diferentes perspectivas, requerimientos y capacidad de negociación. De la misma forma, es necesario comprender que la empresa tiene un conjunto de relaciones con otros actores, que incluyen a la misma población, los organismos no gubernamentales, otras empresas privadas involucradas en la cadena productiva de la empresa, etc. Cada una de ellas constituye un grupo de interés particular y por tanto, la discrepancia de intereses puede constituir también una fuente potencial de conflicto.

Un tercer tema de interés que motiva la investigación es que, como parte de lo verificado en los objetivos anteriores, se requiere la comprensión de los impactos ambientales y socioeconómicos que genera la empresa; entender si es que como externalidades de la actividad económica representan un riesgo para el desarrollo de las comunidades, o si afectan negativamente su calidad de vida, costumbres y entorno, y de ser así conocer las medidas que la empresa, dentro de su política de responsabilidad social, propone como alternativas de solución.

\section{ASPECTOS METODOLÓGICOS}

Durante el trabajo de campo efectuado en el proyecto de investigación se explica que la dife- rencias entre las expectativas de las poblaciones involucradas dentro de la zona de influencia de la mina, así como de la empresa, son fuentes de conflicto (sea por la inexistencia de autoridad del Estado, como por la contraposición de los intereses del binomio empresa - comunidad). Para el caso del distrito de Jangas, a 10 kilómetros de la capital departamental donde se ubica la mina y de algunas localidades aledañas, todas ellas ubicadas en la provincia de Huaraz, en el Departamento de Ancash, se ha verificado que esta fuente de conflicto es permanente. (Ver Gráfico 3).

Para encontrar entonces las raíces del conflicto se busca observar, si desde el punto de vista de los pobladores, la empresa cumple con los compromisos; si la situación económica y social ha mejorado desde el inicio de las operaciones; las expectativas con respecto al corto y mediano plazo sobre el desempeño de la relación empresa y comunidad, así como las relaciones económicas (compras de bienes y servicios y promoción del empleo).

\section{Gráfico 3}

Jangas, ubicación geográfica

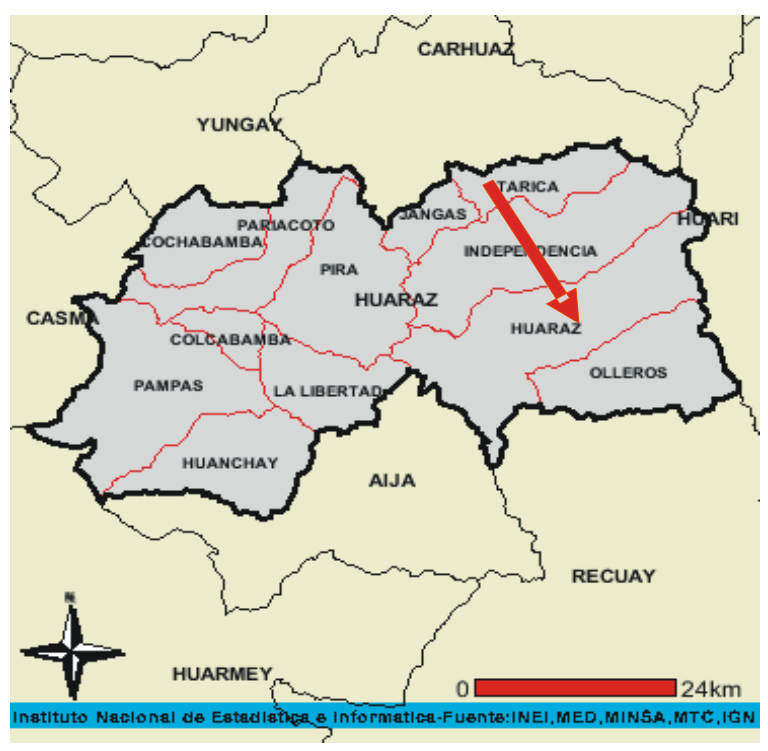

Se visitaron varios lugares: Jangas (Distrito), Pacchac (Quebrada), Atupa (Poblado Menor), Pucauran (Quebrada), Tarica (Poblado Menor), Huanta, San Isidro de Pacollon y San Juan de Pisco. En dichos lugares se realizaron un total de once entrevistas (dos personas en Jangas, una en Huanta, tres en Atupa, cuatro en San Juan de Pisco y al guía). Es notable la reticencia de los pobladores de las localidades de Tarica y San Isidro de Pacollon, quienes se negaron a colaborar 
y proporcionar información con respecto al tema de la mina. Las razones que son causa de esta negativa son múltiples como por ejemplo, el temor a ser identificados como disconformes por parte de la empresa y perder algunos beneficios. La metodología de investigación empleada permite observar fenómenos no conocidos, complejos y dinámicos de la relación entre la empresa y los pobladores.

Se buscaba observar si desde el punto de vista de los pobladores:

a. La empresa cumple con los compromisos.

b. La situación económica y social ha mejorado desde el inicio de las operaciones.

c. Las expectativas con respecto del corto y mediano plazo sobre el desempeño de la relación empresa y comunidad.

d. Las relaciones económicas (compras de bienes y servicios y promoción del empleo).

\section{HALLAZGOS Y RESULTADOS}

Minería en Ancash y la experiencia de Jangas

Pierina, la mina donde opera la Empresa Barrick Misquichilca SA., inicia su producción en 1998 con una inversión de US\$ 275 millones. Actualmente emplea a 430 trabajadores, de los cuales más del $98 \%$ es peruano. Además hay un grupo de 450 campesinos provenientes de zonas aledañas que se desempeñan de manera rotativa en actividades complementarias, tales como reforestación, control de erosión y obras de desarrollo para sus propias comunidades. Es la segunda mina de oro más grande del país.

Ancash ha recibido, gracias a los proyectos mineros establecidos en la zona durante la década de los noventa, ingentes inversiones que se manifiestan además en una dependencia a la actividad minero pero que no ha logrado mayor modificación en los índices de desarrollo humano, como lo refleja el estudio de De Echave y Torres $^{6}$ que presenta los siguientes datos (ver Cuadro 1):

\section{Cuadro 1}

Inversiones vs. Índice de Desarrollo Humano en Ancash (1991-2001)

\begin{tabular}{lcc}
\hline Ancash & $\begin{array}{c}\text { Participación Relativa minera } \\
\text { en \% del PBI (Perú) }\end{array}$ & $\begin{array}{c}\text { Índice de Desarrollo } \\
\text { Humano }\end{array}$ \\
\hline & & (IDH) \\
Año 1991 & 1.4 & 0.503 \\
Año 2001 & 14.2 & 0.577 \\
\hline
\end{tabular}

A su vez, el distrito de Jangas cuya población asciende a 4,345 habitantes, tiene los siguientes indicadores, que representan una reafirmación de lo mencionado sobre el índice del desarrollo humano ${ }^{7}$ (Cuadro 2).

\section{Cuadro 2}

Indicadores de Desarrollo Humano en Jangas.

\begin{tabular}{ll}
\hline Sin agua & $7 \%$ \\
Niños 0-12 & $28 \%$ \\
Sin desaguie & $17 \%$ \\
Desnutrición & $53 \%$ \\
Sin electricidad & $11 \%$ \\
Pobreza (MEF) & $91 \%$ \\
Analfabetismo en mujeres & $42 \%$ \\
Sin instrucción escolar & $18 \%$ \\
\hline
\end{tabular}

Sin embargo, para el año 2006 la comunidad cuenta con una serie de recursos provenientes del Fondo de Compensación Municipal y del Canon Minero además de transferencias (ver Cuadro 3), lo que suma S/. 2774944.00 (casi tres millones de soles), dinero insuficiente para obras de infraestructura física, saneamiento y electrificación a cargo de las autoridades locales.

Cuadro 3

Recursos financieros del distrito de Jangas.

\begin{tabular}{|c|c|c|}
\hline Fuente & Nuevos Soles & Porcentaje \\
\hline $\begin{array}{l}\text { Foncomun- } \\
\text { Foncodes }\end{array}$ & 452802 & $16.32 \%$ \\
\hline $\begin{array}{l}\text { Canon y } \\
\text { otros }\end{array}$ & 2322142 & $83.68 \%$ \\
\hline TOTAL & 2774944 & $100.00 \%$ \\
\hline
\end{tabular}




\section{Percepción de la Población}

En las entrevistas a los diferentes pobladores de las zonas se incidió en las siguientes categorías:

a) Grado de beneficio / perjuicio percibido por las poblaciones con respecto a las operaciones de la minera.

b) Naturaleza de la contribución de las acciones de apoyo social por parte de la minera (obras públicas, empleo, compras a la comunidad).

c) Grado de percepción del compromiso de la minera con la comunidad y su entorno.

En cada una de estas tres categorías las respuestas si bien han sido múltiples pues corresponden a diferentes zonas de influencia de la mina, conservan en esencia el mismo reclamo. Los esfuerzos de la minera se perciben insuficientes por parte de la comunidad, y el punto crítico es la generación de empleo.

Recordemos que, para efectos prácticos, el desarrollo observable, por ejemplo en el índice de desarrollo humano (IDH) de una sociedad está correlacionado con su nivel de ingresos, que depende directamente tanto del grado de especialización y requerimiento de calidad del empleo (demanda) como de la cantidad de pobladores en edad de trabajar (oferta). En Jangas, por ejemplo, la minera ofrece puestos de trabajo en actividades complementarias como limpieza de regadíos, con salarios sumamente bajos. Lo propio se puede decir respecto al apoyo a actividades económicas tradicionales, como por ejemplo, la agricultura. Los pobladores señalan la contaminación y escasez de agua potable. En las entrevistas la población se queja sobre su situación socioeconómica; incluso algunos señalaron que ésta se ha deteriorado.

«La mina hace pequeñas obritas», "ni trabajo nos dan", «la mina da trabajo eventual por tres meses y luego los despiden y contratan a otro grupo», "los trabajos son actividades complementarias como labores de limpieza en los regadíos y con salarios sumamente bajos», «en la construcción de diques en una quebrada de reforzamiento», «en Atupa la empresa ha dado material para la construcción de puentes y otras pequeñas obras», «en otras comunidades han dado apoyo para mejoramiento de viviendas, locales comunales, vías de acceso, etc.»,

son algunas de las respuestas más sentidas de la población.
Como se puede observar es posible que la fuente de discrepancias entre la comunidad y la empresa se sostenga en la diferente percepción de necesidades apremiantes y de los costos sociales del impacto de las operaciones de la empresa sobre el suelo, el agua y la extracción propiamente dicha del mineral.

En el estudio de campo en las comunidades de Jangas, Pacchac, Atupa y Puccauran la población tiene expectativas muy grandes respecto a los beneficios que puede generar la empresa en la zona y al ser zonas del país donde predomina la pobreza (en términos de necesidades básicas, infraestructura y servicios), se perciben altos niveles de insatisfacción. Sus aspiraciones se articulan en el desarrollo de oportunidades económicas (por ejemplo, empleo permanente y directo o compra de productos locales). Este hecho es difícil incluso tomando en cuenta las escasas capacidades técnicas y la baja productividad local. En las entrevistas se observó que entre los pobladores hay descontento porque manifiestan que:

«no se ha recibido ningún tipo de ayuda para la agricultura y la artesanía», $O$ «hemos pedido capacitación para la mejora de la agricultura».

Algunos pobladores, por su parte, señalan entre otros problemas la contaminación o escasez de agua, y que algunas inversiones productivas, como una granja de cerdos o varios biohuertos, han cerrado.

Además, el inicio de actividades en la mina ha generado -como lo manifiesta uno de nuestros entrevistados- el encarecimiento del costo de vida tanto en la propia comunidad como en la capital del departamento, en Huaraz, así como la migración casi forzosa de los pobladores producto, entre otras causas, de la escasez de agua para el riego de los terrenos agrícolas, modificando radicalmente la actividad tradicional.

En opinión del Alcalde de Jangas (elegido para el periodo 2002-2006) en una entrevista, menciona que el apoyo de la mina se ha estado concretando en acciones brindadas a la comunidad en lo relativo a la construcción de obras de infraestructura física; así, se ha suscrito un convenio donde la municipalidad y la empresa aportan 221 mil soles cada una para obras en distintos caseríos en la jurisdicción; sin embargo, las obras tienen poco impacto social, hecho que es ratificado por las manifestaciones de algunos de los entrevistados. 
Otro hecho importante que se extrae como conclusión de la entrevista a las autoridades locales y pobladores de los distritos en estudio, en especial Jangas, es que la empresa minera les informa que dispone de recursos suficientes para apoyar y financiar proyectos, pero que éstos deben ser presentados por la población. El problema es que deben adecuarse a una determinada formalidad y presentar estudios técnicos, cuya elaboración está lejos de las capacidades de los habitantes de la zona. Más aún, es exigencia para la constitución y formulación de alternativas de inversión en infraestructura física la adecuación al Sistema Nacional de Inversión Pública, cuyas exigencias técnicas son especializadas y obligarían -eventualmente- a la contratación de profesionales de fuera para la presentación de los proyectos de inversión.

\section{Conflictos: Paro Regional del 7 de marzo de 2005}

Entre las acciones más críticas ocurridas entre las principales empresas y la población afectada, resalta el Paro Regional del 7 de marzo del 2005 , originado por el desacuerdo de la población con el Fallo del Tribunal Fiscal que le permite a Barrick no pagar impuestos por la ampliación de accionistas de la mina Pierina y cuyos acontecimientos más importantes son ${ }^{8}$ :

- Se estableció una mesa de diálogo presidida por el Alcalde de la Provincia de Huaraz e integrada por representantes de la sociedad civil y colegios profesionales (CIP).

- En el proceso de las negociaciones se presentaron un conjunto de discrepancias, especialmente relacionadas con los temas ambientales. Frente a la serie de problemas para llevar adelante este proceso, solicitaron la intervención, como mediador, del Obispo de la Diócesis de Huaraz.

- Con relación a temas ambientales no hubo un consenso para el proceso del control ambiental, derivado de la indecisión sobre quién financiaría estos pagos. Los representantes del Comité de Lucha no aceptaron que fueran las empresas mineras.

- Frente al problema ambiental, los representantes de la mesa de diálogo acordaron desactivarla y crear dos comisiones técnicas en su reemplazo:

a) Comisión Técnica para el desarrollo del proyecto del Mercado de Abastos de la ciudad de Huaraz, integrada por representantes del
Gobierno Provincial de Huaraz, de los comerciantes y otras instituciones.

b)Comisión Técnica para el desarrollo del proyecto Centro Materno Infantil, integrada por representantes del Hospital de Huaraz, la Dirección Regional de Salud y Colegio de Ingenieros, filial Huaraz.

A la fecha, las Comisiones Técnicas vienen trabajando: en el caso del proyecto del Mercado Mayorista en Huaraz se ha terminado el estudio, pero se han presentado problemas entre los comerciantes sobre la potencialidad del proyecto. Con relación al proyecto Centro Materno Infantil se tiene conocimiento de que hay un avance significativo del mismo.

Los espacios de diálogo que presentan las empresas mineras se dan a través de las organizaciones comunales y su oficina de Relaciones Comunitarias, resaltando la ubicada en el área de influencia de la Unidad Minera Pierina, donde se ha conformado el Comité de Gestión Ambiental de Jangas, integrado por el Alcalde del distrito de Jangas quien actúa como Presidente; Comisión Diocesana de Servicio Pastoral Social de Huaraz (CODISPAS) como Secretaría; el Decano de la Facultad de Ciencias del Ambiente de la Universidad Nacional Santiago Antúnez de Mayolo (UNASAM); Consejo Nacional del Medio Ambiente (CONAM); Distrito de Riego; Comunidades Locales y otros. Este Comité surge como consecuencia de la presencia de la enfermedad conocida como dermatitis y pretende identificar sus causas, por lo que desarrollan acciones, de manera inopinada, relacionadas al monitoreo del agua y aire a través de subcomisiones especializadas ${ }^{9}$.

El 4 de mayo del 2006, un aproximado de 20 comunidades campesinas, caseríos y anexos del distrito de Jangas, convocaron a un paro para protestar por los bajos montos que recibían por jornal diario de la empresa ADECCO (service de la Mina Pierina). La Defensoría del Pueblo supervisó el cumplimiento de funciones por parte de la Policía Nacional del Perú (PNP) y evitó cualquier tipo de enfrentamiento y vulneración al derecho a la libertad personal. Se constató el bloqueo de dos vías de acceso a la Mina Pierina, una por Jangas y otra por la localidad de Palmira.

Se impedía así la entrada y salida de vehículos, y el libre tránsito de los trabajadores de la compañía minera. A las 15:00 horas, la 
Defensoría del Pueblo asistió a una reunión junto con los dirigentes de la compañía minera, aproximadamente 300 comuneros, el Prefecto de Ancash y un sacerdote de la comunidad. Se expresó el siguiente pliego de reclamos:

- Eliminación de los services, exigiendo un contrato directo con la minera y aumento de sueldos.

- Capacitación, entrenamiento e inmediata contratación de electricistas, operadores y otros.

- Apoyo social a las comunidades en cuanto a la construcción de infraestructura, tomándose en cuenta la contratación de adultos mayores.

- Destitución de malos funcionarios y empleados por abusos cometidos en la contratación de personal.

- Disminución del caudal del agua y de los diferentes puntos de captación y la no interrupción del normal caudal del agua, específicamente en la zona de Yarcoc.

- Solicitud de garantías para todos los participantes en el paro.

El día 5 acordaron reunirse en el local de la parroquia el sacerdote, el Prefecto de la Región y un notario público. Los comuneros no participaron, exigían que fuese en otro lugar y con la presencia de las comunidades. La Defensoría del Pueblo exhortó a las partes a llevar a cabo la discusión, apelando a mecanismos que demostrasen transparencia en el proceso. En ese sentido, los representantes de la minera deseaban continuar el diálogo en el lugar neutral acordado. Luego ocurrió un enfrentamiento en el centro poblado Shecta entre la PNP (División de Operaciones Especiales) y miembros del centro poblado, suspendiéndose las negociaciones. Los comuneros habían secuestrado a siete policías, robándoles cuatro fusiles AKM. La consecuencia del enfrentamiento fue un muerto y diecinueve personas heridas (diez comuneros, nueve de la PNP).

Este relato demuestra que las insatisfacciones entre la población local y la empresa han llegado incluso a enfrentamientos con la autoridad, que pueden desvirtuar el propósito inicial de la protesta e incidir en acciones de supuesta reivindicación, derivados no sólo de la influencia de la actividad minera, sino de la postergación que sufren muchas provincias, distritos, centros poblados y comunidades. Otros conflictos, con similares repercusiones registradas durante el periodo de investigación son:
- Tambogrande-Manhattan Minning. Puso en la agenda del debate nacional aspectos relacionados con la viabilidad ambiental, las implicancias económicas y sociales de los proyectos y el inadecuado sistema de participación pública.

Espinar-Billington-Tintaya. Puso de manifiesto la debilidad del sistema de gestión pública que no cuenta con el respaldo de la población, con mecanismos de diálogo que no gozan de la credibilidad necesaria. En el caso de Tintaya, ni el marco legal ni la institucionalidad estatal ni los mecanismos voluntarios de la propia empresa, pudieron crear condiciones adecuadas para poder abordar los elementos centrales que estaban a la base del conflicto.

\section{ANALISIS Y DISCUSIÓN}

\section{Desfase entre inversión minera e inversión social}

La propia naturaleza de la actividad minera supone un desfase entre el comienzo de actividades de exploración y explotación minera en la zona y la generación de utilidades por parte de la empresa. Incluso una vez que una empresa minera genera utilidades, puede pasar más de un año hasta que éstas sean transferidas a la comunidad como canon minero a ser utilizado en proyectos de inversión que se demoran en concretizar, debido al retraso propio del proceso de recaudación tributaria y calendarización del presupuesto público. Ello hace que a nivel local, la población observe que pasan de ocho a quince años en promedio para que se realicen importantes inversiones y obras en su zona, sin constatar resultados que los beneficien directamente (ver Gráfico 4).

Para poder indagar en la problemática del canon minero y vigilar que sus recursos sean invertidos adecuadamente, el gráfico permite observar tres aspectos claves del circuito que recorre el departamento de Ancash:

1. La actividad minera presente, caracterizada por los proyectos de Pierina (Barrick), Antamina y la Minera Santa Luisa, además de otras empresas de mediana y pequeña minería en el departamento de Ancash.

2. Las transferencias que ha recibido hasta mediados del 2005 según la fuente, que como podemos observar, tienen una tendencia creciente. 
3. Los proyectos y obras planificados con dichos recursos para los años indicados, que están caracterizados por obras de infraestructura (desarrollo urbano, educación, saneamiento, agropecuario, etc. ${ }^{10}$.

\section{Inversión social de Barrick}

Barrick tiene un programa de apoyo social cuyo objetivo es lograr el desarrollo sostenible en nueve comunidades campesinas y caseríos vecinos a las operaciones de la mina Pierina, convirtiendo a estas comunidades en unidades económicas de producción y de servicios para lograr el desarrollo autosostenido y la mejora de la calidad de vida. Tiene por estrategia proporcionar asistencia y orientación técnica, así como brindar facilidades y apoyo material. Algunas acciones se observan en la integración vial mediante la construcción de la carretera Jangas - Pierina, mejoramiento de la infraestructura de agua para consumo y riego, ampliación de la infraestructura educativa y sistema de transporte escolar; el otorgamiento de servicios médicos, y desarrollo de productos y mercados.

El programa de inversiones de Barrick se inicia con talleres participativos en las comunidades, a fin de identificar sus potencialidades y fortalezas, en varias líneas de acción, tales como educación, salud y desarrollo productivo. Por ejemplo, en la Comunidad de Andrés Avelino Cáceres de Cuncashca, se ha implementado un
Centro de Producción Agropecuaria para la producción y comercialización de lácteos; además se construyó y mejoró la infraestructura de riego, mejora de pastos y de especies, como complemento a esta actividad ${ }^{11}$. Así mismo se han hecho esfuerzos en educación y salud; en este último aspecto la empresa ha contribuido con la implementación de hospitales y postas médicas, así como la realización de campañas de salud. Asimismo en el campo de la educación ha proporcionado movilidad a los escolares de las diferentes poblaciones a fin de que a concentrar un mayor número de profesores y alumnos en un solo centro educativo, y así lograr que la calidad de la educación mejore.

En el estudio de campo que se efectuó encontramos la mejor obra de infraestructura educativa de la zona. Se trata del colegio Robert Smith, localizado en el poblado menor de Tarica, colegio que está al servicio exclusivo de los hijos de los trabajadores mineros. La empresa también ha realizado en esta misma localidad el asfaltado y alumbrado público. En la capital distrital de Jangas, la empresa ha proporcionado material para la construcción de campos deportivos, el Palacio Municipal, la iglesia y la plazuela.

\section{Mitigación del impacto socio-económico ${ }^{12}$}

Dado que Barrick es consciente de los posibles impactos, tanto positivos como negativos

Gráfico 4

Ciclo de vida de la inversión y del canon minero.

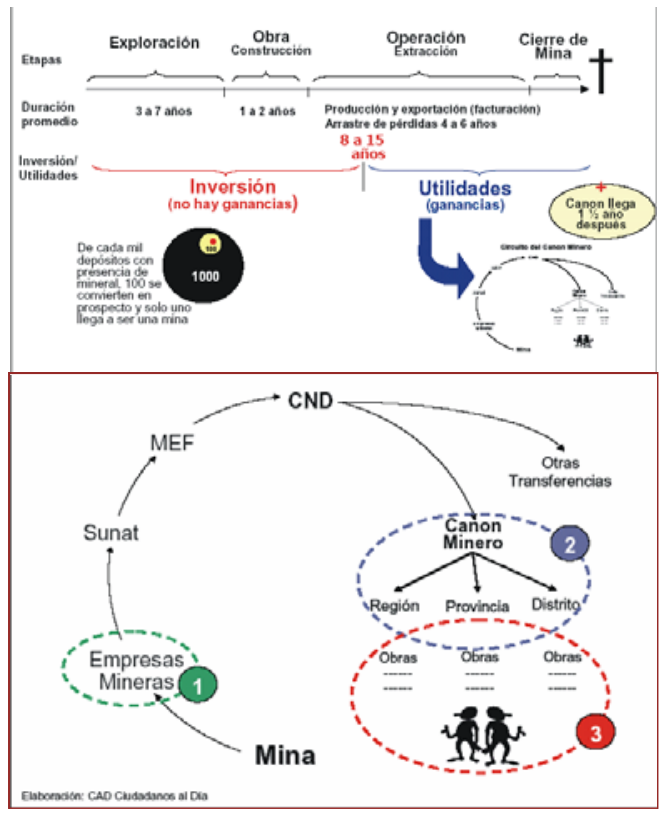


de la minería, se planteó como objetivo alentar un enfoque cordial y respetuoso hacia los habitantes del área, creando así un sentido de participación conjunta además de educar a las comunidades locales acerca de los aspectos positivos y oportunidades ofrecidas por el proyecto por medio de un programa de educación y entrenamiento específicamente diseñado con aquella finalidad.

Barrick, según el Estudio de Impacto Ambiental, indica que prestará especial interés a los problemas de los servicios de infraestructura social que requieren las distintas comunidades y espera promover proyectos diseñados para mejorar las condiciones agrícolas en el área, y mejorar el nivel de vida, especialmente salud y educación de los habitantes locales que viven en el área directamente afectada por el proyecto.

1. Plan de Acción para el Desarrollo Social. Barrick se ha propuesto un plan de desarrollo social que le sirve de guía para mitigar los potenciales impactos sociales y económicos que se asocian a la explotación minera, que tiene los siguientes objetivos:

- Promover el desarrollo económico auto sostenido de los habitantes locales mediante la optimización y mejoría de los recursos locales existentes.

- Introducir tecnología apropiada para mejorar el rendimiento y productividad de sus principales actividades tales como la agricultura y ganadería. Por ejemplo, la empresa contribuye con garantizar el riego de 96 nuevas hectáreas de terreno dedicado a agricultura de consumo, mediante el Canal Chinac del caserío de Canibamba Bajo ${ }^{13}$.

- Mejorar el nivel de vida por medio de programas encaminados a incrementar los niveles de salud y nutricionales, y la promoción tanto de la educación formal a un nivel inicial como de la educación técnica y secundaria. Un ejemplo de ello es la promoción de la III Feria de Orientación Vocacional, donde tres mil alumnos de los centros educativos del departamento recibieron información sobre orientación y posibilidades de estudio ${ }^{14}$.

- Desarrollar un programa de reorganización urbana para optimizar el uso de la infraestructura de servicios básicos. Así tenemos la carretera San Juan de Pasco - Huanja que beneficia a dos mil quinientas personas de centros poblados cercanos a la mina; o también las obras de saneamiento del sistema de desaguie del distrito de Huanta, que incluyen la construcción de pozos sépticos y de percloración, en beneficio de seiscientas personas ${ }^{15}$.

- Proyectar y ejecutar un programa de información, educación y entrenamiento, que incluirá todas las actividades que se ejecutarán dentro del área, de acuerdo con las metas fijadas.

El Plan de Acción para el Desarrollo Social se trazó y se basó durante el periodo en estudio en: los resultados de un estudio de la población local; entrevistas con las autoridades locales; observaciones de las características sociales y culturales locales; sugerencias hechas por funcionarios de Barrick y por consultores especialistas con respecto al desarrollo agrícola, infraestructura urbana y salud; y sugerencias hechas por la Asociación Multidisciplinaria de Investigación y Docencia en Población (AMIDEP) con respecto a consideraciones sociales, económicas y de comunicación social.

2. Desarrollo Económico Autosostenido. Barrick no espera ofrecer empleo directo para toda la gente que vive en el área, ni siquiera para las cabezas de las mil ochocientas familias que viven en el área vecina, ya que solo unos quinientos empleos permanentes resultarán del Proyecto Pierina. No obstante, muchos de estos cargos requieren entrenamiento técnico en prácticas y equipo minero moderno que estará más allá de las capacidades actuales de la población local. Asimismo, la mayoría de las oportunidades de empleo estarán limitadas a la duración prevista del Proyecto, la cual es de aproximadamente quince años.

En vista de estas limitaciones, el Plan de Acción para el Desarrollo Social de Barrick presta atención al mejoramiento de las actividades agrícolas y productivas. El ofrecer ayuda técnica relacionada con la producción agrícola permitirá a los miembros de la población local mejorar sus ingresos y mantener un mejor nivel de vida, inclusive después de que se haya completado el Proyecto. El concepto propuesto en el Plan es el de contribuir al desarrollo integral de las comunidades vecinas, auspiciando actividades sostenibles que ayudarán a mejorar la calidad 
de vida de la población local. Los siguientes programas se emprenderán como un medio de alcanzar esta meta:

- Mejoría de técnicas agrícolas (mejores semillas, introducción de nuevos cultivos).

- Mejoramiento del ganado (mejoría genética, programa para mejoría de pastos, granjas de animales pequeños, programas de salud animal).

- Programa de reforestación (tanto de especies nativas como especies nuevas para el área y árboles frutales).

- Proyecto agrícola para la producción (desarrollo de productos con valor agregado que los haga competitivos en el mercado).

- Programa para la mejoría de la infraestructura económica por medio de la construcción de canales de irrigación, reservorios de agua, andenes de cultivo en las laderas, revolcaderos de ganado, cercas, etc.

3. Mejoría en la Calidad de Vida. El Plan de Acción para el Desarrollo Social incluye las medidas siguientes que pretenden mejorar la infraestructura social y económica básica para buscar un mejor nivel de vida:

- Desarrollo de programas de mejoría urbana y desarrollo de viviendas en las comunidades locales. Mejoramiento del suministro de servicios básicos tales como agua potable, tratamiento de aguas servidas y electricidad.

- Mejoría de la infraestructura de salud y de educación existente mediante la construcción o el reacondicionamiento de centros de salud y/o escuelas en las comunidades que lo requieran, así como el suministro de equipo y provisiones (por ejemplo, equipo para cirugía menor, materiales de enseñanza). Esta actividad se coordinará con organismos estatales tales como el Fondo de Cooperación para el Desarrollo Social (FONCODES). Para evitar la duplicidad de medidas, se completará un estudio de factibilidad y se hará un intento de concentrar estos servicios en comunidades que se convertirán en puntos de convergencia de varios pueblos.

- Construcción de una red de caminos que, además de servir a Barrick, permitirá la comunicación entre las comunidades, las capitales de distrito y la ciudad de Huaraz.
- Respaldo para la modernización del sistema de educación formal en los niveles primario, secundario y técnico, y ayuda a la educación mediante equipos de video, transporte escolar, etc.

- Ayuda para servicios de salud a un nivel regional: Hospital Regional. Se celebrarán convenios especiales con esta finalidad, para optimizar los recursos humanos y materiales de las instituciones de salud existentes. Se prestará especial énfasis en el cuidado general de salud y el tratamiento de enfermedades ocupacionales.

\section{Toma de decisiones de las comunidades y visión de desarrollo}

Para la viabilidad del desarrollo de proyectos mineros, en el paso desde un esquema de compensación hacia una construcción de la visión de desarrollo, el autor José De Echave manifiesta tres aspectos ${ }^{15}$ : el establecimiento de la visión de desarrollo, el análisis estratégico de la comunidad y la formulación de estrategias. El esquema de compensación consiste en la posición pasiva de la comunidad ante la posibilidad de que las empresas estén dispuestas a reconocer las externalidades negativas de sus actividades (la empresa reconoce y compensa, y la comunidad acepta y recibe). Si bien es cierto se trata de un avance en el cumplimiento de ciertos criterios de responsabilidad social, posee muchas limitaciones como soluciones de corto plazo, desigual capacidad de negociación, asimetrías de información, etc.

El paso de un esquema de compensación a uno de visión de desarrollo constituye un desafío para los diferentes actores, en especial de la comunidad que elige el rol de la actividad minera en ese proceso; en cambio el desarrollo comunitario se observa en:

- La visión de desarrollo que se constituye en un marco de referencia para la negociación entre empresa y comunidad, y permite colocar la relación en un contexto superior al de la simple búsqueda de compensaciones puntales.

- El análisis estratégico es el segundo paso de este proceso hacia la visión de desarrollo comunitario. En particular, permite a la comunidad recibir información por parte de la minera y de los demás involucrados, sobre los aspectos globales y particulares de la inversión minera. 
- La construcción de estrategias comunitarias para el desarrollo de la relación con las mineras es el tercer paso, que se puede observar en las siguientes estrategias centrales: fortalecimiento organizativo (creación de instancias y adecuación de la estructura en las comunidades), desarrollo de capacidades (capacitación y formación, transferencia de conocimientos, convenios de colaboración, etc.), y relacionamiento (que es el tipo de estrategia que garantiza mecanismos de solución de conflictos, además de la confluencia de instituciones de apoyo).

El proceso de toma de decisiones comunitarias frente a las operaciones mineras, según el autor citado, busca fortalecer al actor local como un elemento determinante que permite ganar competitividad social y ambiental en la perspectiva de un objetivo de desarrollo local, donde la minería puede jugar un rol complementario y gravitante ${ }^{17}$.

Por otra parte, no debe dejar de pensarse que los recursos naturales son una fuente de desarrollo, y que las capacidades de las comunidades, regiones y países deben desarrollarse en el marco de la responsabilidad compartida de los involucrados. En palabras de Boisier (2006):

\begin{abstract}
«Producir sinergia para el desarrollo de la región a partir de la coordinación de estos actores y opciones supone: a] reunir «cara a cara» $a$ los actores en torno a una «mesa sinergética»; b] evaluar y jerarquizar la contribución de cada opción al desarrollo de la región; cl construir una malla de opciones (no una simple suma); d] obtener compromisos de ejecución; el establecer un mecanismo de seguimiento y retroalimentación. Es fácil imaginar las dificultades de este sencillo ejemplo y es también fácil imaginar la casi imposibilidad de realizar esta coordinación en una situación real, de, digamos, doscientos o más actores, cada uno con una amplia variedad de opciones ${ }^{18}$.
\end{abstract}

La iniciativa privada, sin la legitimidad de los diferentes actores públicos, se convierte en una fuente potencial de conflicto y así lo demuestra la experiencia de la minería en el Perú.

\section{CONCLUSIONES}

1. La actividad minera en el Perú produce y ha producido un conjunto de pasivos ambientales y deterioros sociales. Esta práctica empresarial fue compartida por la gran, pequeña y mediana empresa, tanto nacional como extranjera, a lo largo de la vida republicana, donde predomina el enclave, estructura territorial que caracterizó a la actividad minera; esta estructura exacerba una polarización entre una producción moderna en la explotación de recursos naturales, cuya modalidad de actuación aísla y enfrenta a la empobrecida economía local y regional, generando problemas de depredación de recursos y contaminación ambiental.

2. Esta situación está siendo modificada desde los años 90 con el surgimiento de una nueva generación de prácticas empresariales, cuya visión reúne nuevos elementos estratégicos orientados a poner en práctica programas de desarrollo sustentable con participación de las comunidades, y que corresponden a las nuevas inversiones mineras de origen extranjero, así como a la implantación de empresas filiales de corporaciones transnacionales líderes en la minería. Por ello, para muchas empresas, la competitividad internacional está en relación con la «eliminación de los pasivos ambientales» lo que requiere de la aplicación de nuevas tecnologías de producción «limpias», tales como recuperación de gases y sustancias químicas contaminantes. Asimismo, otra práctica nueva se refiere a la responsabilidad social; no solo involucra a sus trabajadores, sino también a las poblaciones ubicadas en el área de influencia de la mina y de sus instalaciones complementarias o auxiliares, que van desde la infraestructura física, vial, de transporte así como plantas de refinación y otros procesos industriales. Se trata de un enfoque teórico que vincula la sostenibilidad económica empresarial con la responsabilidad social, con la idea de contribuir a la competitividad económica de la zona, con el criterio de que la mina puede trabajar en periodos más o menos extensos (cinco, diez, cincuenta años o más), pero al final se acaba el mineral y la empresa cierra sus operaciones, buscando que exista una dinámica de crecimiento local y desarrollo alternativo a la mina. De la misma forma, se vincula con el desarrollo local que se orienta al desarrollo de las comunidades integrando el reconocimiento de sus posibilidades, recursos, potencialidades y capacidades para conseguir objetivos, y que difiere de la óptica centralista del desarrollo y de la simple división del trabajo regional. 
3. Existe una discrepancia entre el inicio de las operaciones, el inicio de la producción, el pago de las regalías, impuestos y demás transferencias al gobierno central y a las comunidades, y la puesta en marcha de proyectos de inversión (productiva, de infraestructura, social, etc.), lo que en sí constituye una fuente de conflicto estructural que presiona a la empresa, además de la legislación.

4. La compañía minera Barrick señala en sus Planes Estratégicos que desarrolla una política de involucramiento con las comunidades de su área de influencia, para realizar de manera conjunta con ellas acciones de sostenibilidad medio ambiental como control de la erosión, evitar los efectos de los trabajo de movimiento de tierras (deslizamientos, contaminación, control de las fuentes de agua para no perjudicar la actividad productiva de la zona, etc.). De la misma manera, contribuye al desarrollo social y económico con apoyo para la medicación del ganado, obras de educación y vivienda, infraestructura económica como irrigaciones, reservorios, reforestación de la zona, apoyo a la agricultura, etc., así como el impulso hacia la formación de una nueva agricultura de altura, ganadería de alpacas y otros nuevos productos vinculados con la experimentación-aclimatación en viveros y con programas de capacitación. En síntesis, su visión integral es «el desarrollo de las potencialidades socio-económicas de cada comunidad».

5. En el estudio de campo en las comunidades de Jangas, Pacchac, Atupa y Puccauran se aprecia que la población tiene expectativas muy grandes respecto a los beneficios que puede traer la empresa a la zona. Dado que son zonas en donde la pobreza es predominante, en términos de ausencia de infraestructura y servicios para cubrir sus necesidades básicas de educación, salud, saneamiento y agua potable, y otros servicios para sus viviendas, además de alimentación y nutrición; se perciben altos niveles de insatisfacción. Sus aspiraciones se articulan en el desarrollo de oportunidades económicas (empleo permanente y directo en la mina, compra de productos producidos en las comunidades, etc.) lo cual también resulta difícil por la baja productividad local y la ausencia de determinadas capacidades técnicas.

6. Como hemos visto en el presente artículo, se han producidos varias situaciones conflictivas entre empresas mineras y las comunidades de su entorno cercano, así como también conflictos con autoridades y sociedad civil en ámbitos territoriales más amplios (provincias y regiones), como Tintaya, Huaraz, Cajamarca, etc. ¿Qué puede hacer el gobierno? Por un lado, dar un ejemplo con su propia conducta, acelerando el proceso de descentralización y realizando y/o promoviendo inversiones básicas. Además debería clarificar conceptos, delimitar responsabilidades y propiciar espacios de discusión, negociación y concertación, con la participación de todos los actores sociales y económicos involucrados.

7. Una posible alternativa de solución ante la permanente posibilidad de conflicto entre comunidad y empresa, en particular para el caso minero, es el paso de una visión de la responsabilidad social basada en un esquema de compensaciones, hacia un esquema de Visión de Desarrollo, en que se plantee (1) un marco de referencia para la negociación entre empresa y comunidad; (2) un análisis estratégico que permita a la comunidad recibir información de la minera y demás involucrados sobre los aspectos globales y particulares de la inversión minera; y (3) la construcción de estrategias comunitarias como fortalecimiento organizativo, desarrollo de capacidades y de relacio-namiento.

\section{NOTAS}

1 Albuquerque, Francisco. (2003). Teoría y Práctica del Enfoque del Desarrollo Económico Local. Madrid, Consejo Superior de Investigaciones. Instituto de Economía y Geografía.

2 Albuquerque, Francisco. (2004). El enfoque del Desarrollo Económico Local. Buenos Aires, Cuadernos DEL, № 1. Julio, 2004.

3 Aparecido en el Diario Gestión, del 15 de Agosto del 2005.

4 Echave, José y Torres, Víctor. (2005) Hacia una estimación de los Efectos de la Actividad Minera en los índices de Pobreza en el Perú. Lima, Febrero 2005.

5 Iguiñiz, Javier. (2004). Somos Ricos porque somos Pobres. Lima, Fondo Editorial del Congreso de la Republica, Junio 2004.

6 De Echave y Torres, Op cit. P. 86.

7 Información del Fondo para la Cooperación para el Desarrollo Social (FONCODES), 2006, e Instituto Nacional de Estadística e Informática (INEI), 2005.

8 Entrevista a la Dra. Zarela Trinidad de CODISPAS. Diócesis de Huaraz.17 de febrero 2006.

9 Vigila Perú. (2005). Vigilancia de las Industrias Extractivas: Minería. Reporte Regional N 3. Balance 2005 de la Región Ancash - Huaraz. Grupo Propuesta Ciudadana. Marzo del 2006.

10 Boletín CAD. (2005). Canon Minero en Ancash. Ciudadanos al Día, Inversión Minera en Ancash, Mayo 2005. 
11 Tomado de: Barrick, Responsabilidad Social y Ambiental, aparecido en la revista Caretas - Lima, Noviembre del 2005.

12 La presente sección se ha derivado del Estudio de Impacto Ambiental (EIA) presentado por Barrick al Ministerio de Energía y Minas en 1997, donde la empresa expone, entre otras cosas, el Plan de Acción para el Desarrollo Social y que contiene los objetivos y medidas por parte de la empresa.

13 Datos aparecidos en el Boletín: Gente de Pierina Barrica. № 36, octubre del 2004, pp. 8 y 9.

14 En el Boletín: Gente de Pierina Barrica. № 38, octubre del 2004, p. 11

15 En el Boletín: Gente de Pierina Barrica. № 38, octubre del 2004, p. 10

16 De Echave, José. (2001) Construyendo un proceso de toma de decisiones frente a operaciones mineras. Serie Minería y Comunidades. Lima, Acción Solidaria para el Desarrollo (COOPERACCION), p. 132.

17 De Echave, Op. cit, p. 148.

18 Boisier, Sergio. (2006). El Vuelo de una cometa. Una metáfora del desarrollo territorial. Instituto Latinoamericano para el Desarrollo Social. En el Tercer Curso Nacional de Capacitadores para la promoción del Desarrollo Económico Local, organizado por OIT, Swisscontact y COPEME. Noviembre del 2006

\section{BIBLIOGRAFÍA}

Albuquerque, Francisco. (2003). Teoría y Práctica del Enfoque del Desarrollo Económico Local. Madrid, Consejo Superior de Investigaciones.- Instituto de Economía y Geografía.

Albuquerque, Francisco. (2004). El enfoque del Desarrollo Económico Local. Buenos Aires, Cuadernos DEL, № 1. Julio 2004.

Artículo Barrick, Responsabilidad Social y Ambiental. En la revista Caretas. Lima, Noviembre del 2005.

Boisier, Sergio. (2006). El Vuelo de una cometa. Una metáfora del desarrollo territorial. Instituto Latinoamericano para el Desarrollo Social. En el Tercer Curso Nacional de Capacitadores para la promoción del Desarrollo Económico Local, organizado por OIT, Swisscontact y COPEME. Noviembre del 2006
Boletín CAD. (2005). Canon Minero en Ancash. Ciudadanos al Día, Inversión Minera en Ancash, Mayo 2005.

Defensoría del Pueblo. (2006). Reporte $N^{0} 28$. Conflictos sociales conocidos por la Defensoría del Pueblo al 30 de junio de 2006.

Echave, José y Torres, Víctor. (2005). Hacia una estimación de los Efectos de la Actividad Minera en los índices de Pobreza en el Perú. Lima, Febrero 2005.

Echave, José. (2001). Construyendo un proceso de toma de decisiones frente a operaciones mineras. Serie Minería y Comunidades. Lima, Acción Solidaria para el Desarrollo (COOPERACCION).

Estudio de Impacto Ambiental (EIA) presentado por Barrick al Ministerio de Energía y Minas en 1997.

Fondo de Cooperación para el Desarrollo Social (FONCODES). (2006). Información del Fondo para la Cooperación para el Desarrollo Social. Lima. En colaboración con el INEI.

Iguiñiz, Javier. (2004). Somos Ricos porque somos Pobres. Lima, Fondo Editorial del Congreso de la Republica. Junio 2004.

Minera Barrick Misquichilca. (2004). Boletín Informativo: Gente de Pierina. Publicación de la Minera Barrick Misquichilca S.A. del mes de Octubre del 2004, № 36 y 38.

Tello, Mario. (2006). Las teorías del Desarrollo Económico Local y la Teoría y Práctica del Proceso de Descentralización en los países en desarrollo. Lima, Consorcio de Investigación Económico Social. Julio 2006. En: http:// www.pucp.edu.pe/economía/ddd247.pdf

Vigila Perú. (2006). Vigilancia de las Industrias Extractivas: Minería. Reporte Regional № 3: Balance 2005 de la Región Ancash - Huaraz. Marzo del 2006. Grupo Propuesta Ciudadana. 